${ }^{1}$ Первый Московский государственный медииинский университет им. И.М. Сеченова

${ }^{2}$ Московский областной научноисследовательский клинический институт им. М.Ф. Владимирского

${ }^{3}$ Федеральное

медико-биологическое агентство России

${ }^{4}$ Научно-

исследовательский институт скорой помоши

им. Н.В. Склифосовского

\section{Факторы риска и скрининг гепатоцеллюлярного рака после эрадикации вируса гепатита C}

Адрес для переписки: Алексей Олегович Буеверов, bcl72@yandex.ru

Для цитирования: Буеверов А.О., Богомолов П.О., Сюткин В.Е. Факторы риска и скрининг гепатоцеллюлярного рака после эрадикации вируса гепатита С // Эффективная фармакотерапия. 2021. Т. 17. № 28. С. 54-60.

DOI 10.33978/2307-3586-2021-17-28-54-60

Гепатоцеллюлярный рак (ГЦР) обычно развивается на фоне хронического заболевания печени. До недавнего времени наиболее частой этиологией ГЦР было инбицирование вирусом гепатита С (HCV). Появление противовирусных препаратов прямого действия (ПППД) стало большим прорывом в течении HCV-инфекции. Устойчивый вирусологический ответ теперь может быть достигнут почти у всех пролеченных пациентов, даже у лиц с высоким риском ГЦР, в первую очередь с ииррозом печени. Вместе с тем постепенно стали накапливаться сообщения о сохранении риска злокачественной трансформации после успешной терапии ПППД. Одновременно со снижением бремени иирроза, обусловленного HCV, резко возросла этиологчческая роль неалкогольной жировой болезни печени (НАЖБП). Более того, у значительной части пациентов с НАЖБП ГЦР формируется на доцирротической стадии. Эти изменения в этиологии и эпидемиологии ГЦР предполагают ревизию тактики ведения больных.

Ключевые слова: гепатоцеллюлярный рак, вирус гепатита С, факторы риска, противовирусные препараты прямого действия, неалкогольная жировая болезнь печени

$\Gamma$ епатоцеллюлярный рак (ГЦР) - пятый по распространенности и второй по частоте летальных исходов рак в мире [1]. Основным фактором риска развития опухоли является цирроз печени (ЦП), независимо от его этиологии [2]. Эпидемиология ГЦР характеризуется географической неоднородностью: большинство случаев в Азии и Африке связано с вирусом гепатита B (HBV), в то время как вирус гепатита $\mathrm{C}(\mathrm{HCV})$ представляет собой основной фактор риска в западных странах. У пациентов с ЦП и активной инфекцией $\mathrm{HCV}$ годовой риск ГЦР достигает 3\% [3]. Противовирусные препараты прямого действия (ПППД) произвели революцию в лечении $\mathrm{HCV}$-инфекции благодаря высочайшей эффективности и безопасности и позволяют использовать ее даже у пациентов с декомпенсированным ЦП [4]. Устойчивый вирусологический ответ (УВО) снижает риск развития ЦП, а в некоторых случаях обусловливает его регресс $[5,6]$. У пациентов с декомпенсированным циррозом, достигших УВО, наблюдалось снижение баллов по модели терминальной стадии заболевания печени (MELD) и градиента печеночного венозного давления [7]. Более того, достижение УВО снижает как общую смертность, так и смертность от патологии печени [8]. Однако по мере увеличения пула вылеченных пациентов стали накапливаться сообщения о сохранении риска злокачественной трансформации после успешной терапии ПППД $[9,10]$. При этом снижение бремени хронической HCV-инфекции выдвинуло на первый план новую проблему: прогрессирующее увеличение распространенности неалкогольной жировой болезни печени (НАЖБП), занявшей второе место в числе причин включения в лист ожидания трансплантации печени [11].

\section{Факторы риска развития ГЦР у больных HCV-инфекцией}

Хроническое воспаление

Персистирующее воспаление повышает уровень активных форм кислорода, повреждающих гепатоциты как на генетическом, так и на клеточном уровне, что в конечном итоге приводит к их гибели. Модифицированный $\mathrm{HCV}$ иммунный ответ стимулирует компенсаторную регенерацию печени, что способству- 
ет хромосомной нестабильности и соответственно патологическим изменениям генома. Эти изменения в свою очередь стимулируют неопластическую трансформацию гепатоцитов и пролиферацию злокачественных клонов [12].

\section{Гепатит В}

Инфекция HBV, наряду с HCV, относится к числу наиболее распространенных этиологических факторов хронических заболеваний печени и ГЦР. Реактивация HBV после или во время клиренса HCV-инфекции на фоне терапии ПППД представляет собой отдельную проблему, требующую дополнительных исследований для определения тактики. В качестве метода профилактики реактивации HBV после или во время лечения хронического гепатита С рассматривается возможность применения аналогов нуклеозидов $[13,14]$. Важным наблюдением является значительный риск ГЦР у этих больных после достижения устойчивого вирусологического ответа, особенно при наличии выраженного фиброза и других факторов риска (злоупотребление алкоголем, ожирение, сахарный диабет) [14]. Большинство случаев реактивации HBV, проявляющейся значительным повышением активности трансаминаз и уровня HBV ДНК, описаны через 4-12 недель терапии ПППД [15].

\section{Неалкогольная жировая болезнь печени}

Распространенность НАЖБП, чаще всего являющейся компонентом метаболического синдрома, достигает 30-40\% среди взрослого населения [16]. Частота ГЦР у этих пациентов составляет $0,04-0,3 \%$ в год при неалкогольном стеатогепатите (НАСГ) без выраженного фиброза до 2,6\% в год при ЦП [17-22]. Подробнее роль НАЖБП в генезе ГЦР будет рассмотрена ниже.

\section{Метилирование ДНК}

В печеночных биоптатах больных хронической $\mathrm{HCV}$-инфекцией выявлены множественные метилированные гены-супрессоры. Промоторы метилированных супрессоров чаще обнаруживаются в ткани печени, инфицированной $\mathrm{HCV}$, по сравнению с инфицированием другими гепатотропными вирусами [23]. Кроме того, эти промоторы чаще встречаются при ЦП по сравнению с хроническим гепатитом [24]. Накоплены сведения, что гены метилированных опухолевых супрессоров в инфицированной $\mathrm{HCV}$ ткани печени хронологически предшествуют возникновению ГЦР [25].

\section{Оккультная $\mathrm{HCV}$-инфекция}

Оккультная HCV-инфекция определяется как присутствие HCV РНК в клетках печени или мононуклеарных клетках периферической крови в отсутствие HCV РНК в сыворотке [26, 27]. Данные об этой форме инфекции противоречивы: с одной стороны, РНК вируса иногда может быть обнаружена в гепатоцитах и мононуклеарах, с другой - остается неясной ее способность к репликации и тем более иммуноопосредованному повреждению клеток. M.M. Yousif и соавт. установили значительную распространенность оккультной инфекции HCV после лечения ПППД [27]. При этом, несмотря на успешный клиренс вируса, у отдельных пациентов продолжается прогрессирование заболевания до ГЦР, возможно обусловленное $\mathrm{HCV}[28,29]$. Несмотря на то что ПППД более эффективно элиминируют HCV по сравнению с интерферон-содержащими схемами, они, по-видимому, активны против сывороточного, но не всегда против внутриклеточного вируса. Следовательно, репликация потенциально опухолевых клонов продолжается в условиях подавленного воспаления [30]. Соответственно можно предположить, что экзогенный интерферон а обладает защитным эффектом в отношении ГЦР, поскольку он активирует иммунные клетки с проапоптотической и противоопухолевой активностью. E. Serti и соавт. выдвинули гипотезу о том, что после эрадикации HCV с помощью ПППД введение интерферона в схему лечения может реактивировать иммунный ответ против опухолевых клонов [31]. Вместе с тем ГЦР может развиваться и после эрадикации вируса на фоне интерферон-содержащих схем с частотой 2,3-8,8\% [32].

\section{ГЦР и препараты прямого противовирусного действия}

Проведенные ранее проспективные исследования терапии на основе интерферона а показали, что успешное лечение значимо ассоциировано с подавлением печеночного канцерогенеза [33, 34]. Метаанализ 12 исследований продемонстрировал, что достижение УВО на интерферон-содержащих схемах обусловливало снижение риска ГЦР на 76\% [34]. В то же время в ранних работах по оценке пост-эффекта ПППД было высказано опасение, что индуцированный ими УВО не снижает частоту возникновения ГЦР и ассоциирован с высоким риском раннего рецидива у леченных по поводу ГЦР больных. Так, под наблюдением F. Conti и соавт. находились 344 пациента с ЦП, получавших лечение ПППД в течение 24 недель; ГЦР выявлен у 9 (3,2\%) из 285 пациентов, рецидив ГЦР диагностирован у 17 (28,8\%) из 59 ранее получавших лечение пациентов. В качестве независимых факторов риска авторами отмечены класс цирроза по Чайлду - Пью и ГЦР в анамнезе [9].

Напротив, результаты нескольких крупных когортных исследований позволили прийти к заключению, что УВО, индуцированный ПППД, обусловливает снижение риска ГЦР. В частности, в ретроспективное исследование G.N. Ioannou и соавт. было включено более 60000 ветеранов США с HCV, получавших противовирусную терапию в период с 1999 по 2015 г., включая терапию ПППД, схемы на основе интерферона а и комбинированные схемы [35]. После корректировки исходных характеристик пациенты с УВО, индуцированным ПППД, продемонстрировали снижение риска ГЦР на $71 \%$ по сравнению с неэффективным лечением. Кроме того, снижение риска ГЦР у достигших УВО было одинаковым вне зависимости от применяемой схемы, и это позво- 
ляет предположить, что печеночный канцерогенез подавляет именно достижение эрадикации HCV. Систематический метаанализ 26 обсервационных исследований (17 - интерферон $\alpha, 9$ - ПППД) показал более высокую частоту ГЦР у пациентов с УВО, индуцированным ПППД, по сравнению с индуцированным интерфероном (2,96 из 100 пациентолет и 1,14 из 100 пациенто-лет соответственно), но пациенты, получавшие ПППД, были старше и наблюдались менее продолжительное время [36]. Метарегрессионный анализ после корректировки с учетом продолжительности исследования и возраста не выявил связи терапии ПППД с более высокой частотой возникновения ГЦР по сравнению с интерферон-содержащими схемами. Z. Mariño и соавт. сообщили о 3,73\%-м на 1000 человеко-лет риске развития ГЦР у 1123 пациентов с ЦП, получавших ПППД, в течение среднего периода наблюдения 19,6 месяца [37]. Риск предсказуемо оказался выше у пациентов без УВО по сравнению с достигшими УВО, а также с более тяжелым заболеванием (класс В или С по Чайлду - Пью, высокая степень жесткости печени, наличие клинически значимой портальной гипертензии). Таким образом, имеются основания полагать, что наиболее важным фактором, определяющим снижение риска ГЦР, служит эрадикация $\mathrm{HCV}$, независимо от способа, которым последняя была достигнута.

Более спорным до недавнего времени являлся вопрос о повышенном риске рецидива опухоли после ранее проведенной резекции или радиочастотной абляции на фоне достижения УВО. В частности, рецидив ГЦР диагностирован у 16 (27,6\%) из 58 пациентов в испанской популяции [38] и у 17 (28,8\%) из 59 в итальянском исследовании [9] в течение среднего периода наблюдения шесть месяцев. Однако небольшой размер групп, отсутствие контрольной группы и короткая средняя продолжительность наблюдения служили ограничивающими факторами, не позволяющими сделать окончательные выводы. В двух крупных контролируемых исследованиях, а также в одном анализе с поправкой на предрасположенность не сообщалось об увеличении частоты рецидивов ГЦР у пациентов с адекватно проведенным лечением ПППД по сравнению с нелечеными пациентами $[39,40]$.

В уже упомянутом метаанализе с метарегрессией 17 исследований рецидивов ГЦР после терапии ПППД и интерфероном а не обнаружено различий в частоте рецидивирования [36]. Аналогично анализ, проведенный в Японии, также не показал существенной разницы в частоте рецидивов ГЦР между пациентами, получавшими разные схемы терапии [41]. Предсказуемо, что кумулятивная частота рецидивов у достигших УВО была значительно ниже, чем в подгруппе без УВО, в обеих группах лечения. Веские доказательства против связи терапии ПППД с рецидивом ГЦР получены в результате взвешенного по шкале предрасположенности анализа 149 кандидатов на трансплантацию печени c HCV-инфек- цией и ГЦР с первоначальным полным ответом на локорегиональную терапию [42]. Применение ПППД было ассоциировано не с повышенным риском рецидива ГЦР, а скорее со снижением риска исключения из списка ожидания из-за прогрессирования опухоли или смерти [3]. Таким образом, полученные данные свидетельствуют о значительном преобладании пользы от применения ПППД, в том числе в этой особой группе.

\section{Скрининг ГЦР после достижения УВО}

Как отмечено выше, успешная противовирусная терапия хронической $\mathrm{HCV}$-инфекции снижает, но не устраняет риск ГЦР. Поэтому после достижения УВО необходимо продолжать наблюдение за пациентами не только с ЦП (F4), но и с выраженным фиброзом (F3) $[17,43]$. Несмотря на эти рекомендации, анализ экономической эффективности показал, что скрининг ГЦР вряд ли окажется рентабельным после достижения УВО у пациентов с выраженным фиброзом, тогда как ежегодные либо двухгодичные обследования, безусловно, необходимы больным на цирротической стадии [44]. Остается спорным вопрос, приводит ли регрессия фиброза к снижению риска ГЦР и соответственно можно ли прекратить наблюдение за ними. Так, R. D'Ambrosio и соавт. наблюдали за небольшой группой пациентов с HCV, получавших схемы на основе интерферона а в течение почти восьми лет после УВО, но при этом не смогли доказать отчетливого влияния регрессии фиброза в отношении возникновения ГЦР [45].

В ряде исследований предприняты попытки определить факторы риска ГЦР после терапии ПППД. По-видимому, наиболее сильным предиктором является отсутствие УВО [6, 46, 47]. В трехлетнем продольном катамнестическом исследовании 565 пациентов с ЦП в качестве предрасполагающих факторов развития ГЦР de novo были установлены мужской пол, сахарный диабет и плотность печени. При этом только сахарный диабет оказался независимым предиктором рецидива ГЦР после проведенного лечения [47]. Наблюдение в течение одного года за выборкой из почти 2000 пациентов продемонстрировало, что с ГЦР ассоциированы возраст старше 50 лет и наличие варикозно расширенных вен пищевода [48].

Напротив, больные с числом тромбоцитов $>110$ тыс/мкл и плотностью печени $<25$ кПа имеют очень низкую вероятность развития ГЦР и им не требуются частые визиты к врачу [49]. Многофакторный регрессионный анализ Кокса, основанный на проспективно собранных данных из Италии, показал, что уровень альбумина $<3,5$ мг/дл и количество тромбоцитов $<120$ тыс/мкл, наряду с отсутствием УВО, были независимо связаны с более высоким риском развития ГЦР [50]. Дополнительный важный фактор риска печеночного канцерогенеза - метаболический синдром и тесно ассоциированная с ним НАЖБП, значение которых будет рассмотрено ниже $[17,51,52]$. Таким пациентам 
необходимы проведение скрининга ГЦР и оценка скорости прогрессирования заболевания после достижения УВО, о чем они должны быть своевременно информированы.

Таким образом, оценка риска возникновения ГЦР важна для разработки адекватной стратегии скрининга. G.N. Ioannou и соавт. разработали и апробировали модель прогнозирования риска ГЦР с использованием исходных характеристик до начала противовирусного лечения [35, 46]. В выделенных ими четырех подгруппах заболеваемость ГЦР была наиболее высока в подгруппе с ЦП и без УВО и самой низкой у пациентов без цирроза с УВО. Возраст, количество тромбоцитов, соотношение аспартатаминотрансферазы/аланинаминотрансферазы и сывороточный уровень альбумина также имели важное прогностическое значение, в то время как пол, этническая принадлежность, генотип $\mathrm{HCV}$, индекс массы тела, гемоглобин и МНО вносили существенно меньший вклад. Стратегия скрининга, основанная на модели риска, продемонстрировала большую рациональность по сравнению с тотальным скринингом либо отсутствием такового.

\section{Роль НАЖБП в генезе ГЦР}

Всемирная эпидемия ожирения в последние десятилетия неуклонно расширяет свою экспансию, что в свою очередь обусловливает повышение заболеваемости НАЖБП, поражающей, по разным данным, от 13 до $33 \%$ взрослого населения $[16,19,53]$. НАЖБП сегодня - наиболее часто наблюдающееся хроническое заболевание печени в западных странах [19]. В 2016 г. метаанализ 729 исследований (размер выборки более восьми миллионов субъектов из 22 стран) показал, что глобальная распространенность НАЖБП составляет $25,2 \%$ с наиболее высокой на Ближнем Востоке $(31,8 \%)$ и в Южной Америке (30,5\%), а наиболее низкой в Африке (13,5\%). Распространенность подтвержденного биопсией неалкогольного стеатогепатита среди пациентов с НАЖБП варьировалась от 6,7-29,9\% при случайных биопсиях до 60,7-69,3\% у тех больных, которым биопсия выполнялась при подтвержденном диагнозе НАЖБП [53].

Широкая вариабельность течения НАЖБП служит основанием для поиска факторов риска, определяющих скорость ее прогрессирования и вероятность развития ЦП и ГЦР. Среди них наиболее хорошо изучены компоненты метаболического синдрома - ожирение, инсулинорезистентность, сахарный диабет 2-го типа, дислипидемия, артериальная гипертензия. Можно с уверенностью утверждать, что НАЖБП, наблюдающаяся в разных странах у 20-40\% населения, является мультиорганным заболеванием, тесно сопряженным с сердечно-сосудистой, эндокринной, почечной патологией, а также злокачественными опухолями $[16,19,20,49,53]$.

Поиск генов, ассоциированных с более агрессивным течением НАЖБП, продолжается на протяжении почти двух десятилетий, но подтвержденные результаты удалось получить лишь для пататиноподобного фосфолипазного домена 3 (PNPLA3) I148M. Выяснилось, что данный аллель коррелирует не только с тяжестью заболевания, но и с риском развития ГЦР [54, 55]. Вместе с тем позже появились сообщения, что трансмембранный ген 2 суперсемейства 6 (TM6SF2) является еще одним модификатором заболевания, способствуя прогрессированию ЦП и ГЦР [56]. Более того, исследование В.К. Коо и соавт. показало, что варианты риска PNPLA3 и TM6SF2 обладают аддитивным влиянием на прогрессирование фиброза при НАСГ [57].

A.J. Walker и соавт. опубликовали систематический обзор литературы для выявления генетической предрасположенности к ГЦР пациентов, инфицированных HCV. Из 137 генов или их комбинаций для 17 была доказана сильная ассоциация, для 37 - ассоциация средней силы, для 56 - слабая либо с ограниченной доказательной базой; для 27 генов ассоциация установлена не была. Наиболее веские доказательства связи с ГЦР получены для генов интерферона $\lambda 3 / 4$, фактора некроза опухоли а и белка-3, содержащего домен папатиноподобной фосфорилазы (адипонутрина). Авторы обзора подчеркивают важность расшифровки механизмов, лежащих в основе этих взаимоотношений, и их связь с внешними факторами риска ГЦР, а также ответом на терапию [58].

Вероятность возникновения ГЦР достоверно коррелирует со стадией фиброза [21]. У пациентов с нецирротической НАЖБП частота случаев ГЦР составляла 0,04 на 1000 при низком значении индекса FIB-4 и 0,39 на 1000 при его высоких значениях; при ЦП риск повышался до 4,82-13,55 на 1000 [22]. При этом в исследовании S. Mittal и соавт. около $13 \%$ случаев ГЦР обнаруживались в нецирротической печени [59]. Хотя ГЦР при НАЖБП наблюдается реже, чем при хронических гепатитах В или С, высокое абсолютное число таких пациентов во всем мире диктует необходимость поиска решения проблемы. Частота ГЦР, ассоциированного с НАЖБП, увеличивается на 9\% ежегодно [53]. В большом ретроспективном когортном исследовании (2004-2009), включавшем около 5000 больных ГЦР и 15000 пациентов контрольной группы, пациенты с ГЦР на фоне НАЖБП были на момент установления диагноза старше и характеризовались меньшей продолжительностью жизни по сравнению с пациентами с ГЦР на фоне вирусных гепатитов; одногодичная смертность составила для НАЖБП 61,2\%, для гепатита С - 51,3\%, для гепатита В - 43,7\% [18]. Следует учитывать, что у пациентов с НАЖБП выше риск смерти от внепеченочных причин, в первую очередь сердечно-сосудистых.

Ультразвуковое исследование (УЗИ) органов брюшной полости представляет собой исследование первой линии для скрининга ГЦР ввиду относительно невысокой стоимости и отсутствия лучевой нагрузки. В популяции больных ЦП чувствительность УЗИ составляет 58-89\% при специфичности, превышающей 90\% [60]. Однако избыточная подкожно-жировая клетчатка и печеночный стеатоз обусловливают 
ослабление ультразвукового сигнала и ухудшают визуализацию глубоких структур печени. Так, в исследовании 941 пациента с ЦП 20\% ультразвуковых исследований были сочтены неадекватными для исключения ГЦР. Цирроз, обусловленный НАЖБП, и повышенный индекс массы тела идентифицированы как независимые факторы риска невалидных результатов исследования [61]. Использование других методов лучевой диагностики, таких как компьютерная и магнитно-резонансная томография, ограничено низкой экономической эффективностью ввиду обширности популяции риска.

Стеатоз печени также влияет на выбор метода лечения ГЦР и риск осложнений. Вероятность осложнений резекции печени у пациентов с НАЖБП на фоне метаболического синдрома без выраженного фиброза аналогична профилю риска у пациентов с ЦП [62]. У этих пациентов обычно имеются сопутствующие заболевания, такие как ожирение, дислипидемия, артериальная гипертензия, сахарный диабет, ишемическая болезнь сердца, хроническая болезнь почек, которые способствуют ухудшению прогноза.

\section{Заключение}

Высочайшая эффективность терапии HCV-инфекции ПППД в сочетании с отличным профилем безопасности ставит новые вопросы перед врачами. Появление возможности лечения пациентов с некомпенсированным ЦП и сочетанной патологией привело к росту абсолютного числа случаев ГЦР - больные стали доживать до рака, не умирая от осложнений цирроза. Снижение риска канцерогенеза в первую очередь ассоциировано с УВО. На сегодняшний день не получено доказательств различий частоты возникновения или рецидивов ГЦР на фоне лечения ПППД или интерфероном $\alpha$. При наличии ЦП после достижения УВО рекомендуется пожизненное наблюдение; исключение может быть сделано для больных с доказанным регрессом фиброза. Экономически целесообразно стратифицировать пациентов по факторам риска, хотя эта стратегия требует подтверждения результатами новых исследований. Особое внимание уделяется НАЖБП как важнейшей причине печеночного канцерогенеза в экономически развитых странах, в том числе у больных на доцирротической стадии.

\section{Литература}

1. Akinyemiju T., Abera S., Ahmed M. et al. The burden of primary liver cancer and underlying etiologies from 1990 to 2015 at the global, regional, and national level: results from the global burden of disease study 2015 // JAMA Oncol. 2017. Vol. 3. № 12. P. 1683-1691.

2. West J., Card T.R., Aithal G.P., Fleming K.M. Risk of hepatocellular carcinoma among individuals with different aetiologies of cirrhosis: a population-based cohort study // Aliment. Pharmacol. Ther. 2017. Vol. 45. № 7. P. 983-990.

3. Frazzoni L., Sikandar U., Metelli F. et al. Hepatocellular carcinoma recurrence after hepatitis C virus therapy with direct-acting antivirals. A systematic review and meta-analysis // J. Clin. Med. 2021. Vol. 10. № 8. P. 1694.

4. Baumert T.F., Berg T., Lim J.K., Nelson D.R. Status of direct-acting antiviral therapy for hepatitis C virus infection and remaining challenges // Gastroenterology. 2019. Vol. 156. № 2. P. 431-445.

5. Bachofner J.A., Valli P.V., Kröger A. et al. Direct antiviral agent treatment of chronic hepatitis C results in rapid regression of transient elastography and fibrosis markers fibrosis-4 score and aspartate aminotransferase-platelet ratio index // Liver Int. 2017. Vol. 37. № 3. P. 369-376.

6. Rinaldi L., Nevola R., Franci G. et al. Risk of hepatocellular carcinoma after HCV clearance by direct-acting antivirals treatment: predictive factors and role of epigenetics // Cancers (Basel). 2020. Vol. 12. № 6. E1351.

7. Foster G.R., Irving W.L., Cheung M.C. et al. Impact of direct acting antiviral therapy in patients with chronic hepatitis C and decompensated cirrhosis // J. Hepatol. 2016. Vol. 64. № 6. P. 1224-1231.

8. Backus L.I., Belperio P.S., Shahoumian T.A., Mole L.A. Impact of sustained virologic response with direct-acting antiviral treatment on mortality in patients with advanced liver disease // Hepatology. 2019. Vol. 69. № 2. P. $487-497$.

9. Conti F., Buonfiglioli F., Scuteri A. et al. Early occurrence and recurrence of hepatocellular carcinoma in HCV-related cirrhosis treated with direct-acting antivirals // J. Hepatol. 2016. Vol. 65. № 4. P. 727-733.

10. Cardoso H., Vale A.M., Rodrigues S. et al. High incidence of hepatocellular carcinoma following successful interferon-free antiviral therapy for hepatitis C associated cirrhosis // J. Hepatol. 2016. Vol. 65. № 5. P. 1070-1071.

11. Noureddin M., Vipani A., Bresee C. et al. NASH leading cause of liver transplant in women: updated analysis of indications for liver transplant and ethnic and gender variances // Am. J. Gastroenterol. 2018. Vol. 113. № 11. P. 1649-1659.

12. Hoshida Y., Fuchs B.C., Bardeesy N. et al. Pathogenesis and prevention of hepatitis C virus-induced hepatocellular carcinoma // J. Hepatol. 2014. Vol. 61. № 1. P. 79-90.

13. Pisaturo M., Macera M., Alessio L. et al. Hepatitis B virus (HBV) reactivation following pharmacological eradication of hepatitis C virus (HCV) // Viruses. 2019. Vol. 11. № 9. P. 850.

14. Huang C.F., Yu M.L. Unmet needs of chronic hepatitis $\mathrm{C}$ in the era of direct-acting antiviral therapy // Clin. Mol. Hepatol. 2020. Vol. 26. № 3. P. 251-260.

15. Serper M., Forde K.A., Kaplan D.E. Rare clinically significant hepatic events and hepatitis B reactivation occur more frequently following rather than during direct-acting antiviral therapy for chronic hepatitis C: data from a national US cohort // J. Viral. Hepat. 2018. Vol. 25. № 2. P. 187-197. 
16. Драпкина О.М., Буеверов А.О. Неалкогольная жировая болезнь печени как мультидисциплинарная патология. М.: Видокс, 2019. С. 104.

17. Chalasani N., Younossi Z., Lavine J.E. et al. The diagnosis and management of nonalcoholic fatty liver disease: practice guidance from the American Association for the Study of Liver Diseases // Hepatology. 2018. Vol. 67. № 1. P. 328-357.

18. Younossi Z.M., Otgonsuren M., Henry L. et al. Association of nonalcoholic fatty liver disease (NAFLD) with hepatocellular carcinoma (HCC) in the United States from 2004 to 2009 // Hepatology. 2015. Vol. 62. № 6. P. 1723-1730.

19. Younossi Z.M. Non-alcoholic fatty liver disease - a global public health perspective // J. Hepatol. 2019. Vol. 70 . № 3. P. 531-544.

20. Patel Y.A., Gifford E.J., Glass L.M. et al. Risk factors for biopsy-proven advanced non-alcoholic fatty liver disease in the Veterans Health Administration // Aliment. Pharmacol. Ther. 2018. Vol. 47. № 2. P. 268-278.

21. Angulo P., Kleiner D.E., Dam-Larsen S. et al. Liver fibrosis, but no other histologic features, is associated with long-term outcomes of patients with nonalcoholic fatty liver disease // Gastroenterology. 2015. Vol. 149. № 2. P. 389-397.e10.

22. Kanwal F., Kramer J.R., Mapakshi S. et al. Risk of hepatocellular cancer in patients with non-alcoholic fatty liver disease // Gastroenterology. 2018. Vol. 155. № 6. P. 1828-1837.e2.

23. Nishida N., Nagasaka T., Nishimura T. et al. Aberrant methylation of multiple tumor suppressor genes in aging liver, chronic hepatitis, and hepatocellular carcinoma // Hepatology. 2008. Vol. 47. № 3. P. 908-918.

24. Gao D.Y., Zhang X.X., Hou G. et al. Assessment of specific antibodies to F protein in serum samples from Chinese hepatitis C patients treated with interferon plus ribavirin // J. Clin. Microbiol. 2008. Vol. 46. № 11. P. 3746-3751.

25. Toraih E.A., Ellawindy A., Fala S.Y. et al. Oncogenic long noncoding RNA MALAT1 and HCV-related hepatocellular carcinoma // Biomed Pharm. 2018. Vol. 102. P. 653-669.

26. Austria A., Wu G.Y. Occult hepatitis C virus infection: a review // J. Clin. Transl. Hepatol. 2018. Vol. 6. № 2. P. $155-160$.

27. Yousif M.M., Elsadek Fakhr A., Morad E.A. et al. Prevalence of occult hepatitis C virus infection in patients who achieved sustained virologic response to direct-acting antiviral agents // Infez. Med. 2018. Vol. 26. № 3. P. 237-243.

28. Manickam C., Reeves R.K. Silent damage? Occult HCV replication and histological disease may occur following apparent HCV clearance // EBioMedicine. 2019. Vol. 47. P. 12-13.

29. Warzyszyńska K., Jonas M., Wasiak D. et al. Accelerated hepatocellular carcinoma recurrence rate after postoperative direct-acting antivirals treatment - preliminary report // Clin. Exp. Hepatol. 2017. Vol. 3. № 4. P. 194-197.

30. Hengst J., Strunz B., Deterding K. et al. Nonreversible MAIT cell-dysfunction in chronic hepatitis C virus infection despite successful interferon-free therapy // Eur. J. Immunol. 2016. Vol. 46. № 9. P. 2204-2210.

31. Serti E., Park H., Keane M. et al. Rapid decrease in hepatitis $C$ viremia by direct acting antivirals improves the natural killer cell response to IFNa // Gut. 2017. Vol. 66. № 4. P. 724-735.

32. Hiramatsu N., Oze T., Takehara T. Suppression of hepatocellular carcinoma development in hepatitis $\mathrm{C}$ patients given interferon-based antiviral therapy // Hepatol. Res. 2015. Vol. 45. № 2. P. 152-161.

33. Bruix J., Poynard T., Colombo M. et al. Maintenance therapy with peginterferon alfa-2b does not prevent hepatocellular carcinoma in cirrhotic patients with chronic hepatitis C // Gastroenterology. 2011. Vol. 140. № 7. P. 1990-1999.

34. Morgan R.L., Baack B., Smith B.D. et al. Eradication of hepatitis C virus infection and the development of hepatocellular carcinoma: a meta-analysis of observational studies // Ann. Intern. Med. 2013. Vol. 158. № 5. Pt. 1. P. 329-337.

35. Ioannou G.N., Green P.K., Beste L.A. et al. Development of models estimating the risk of hepatocellular carcinoma after antiviral treatment for hepatitis C // J. Hepatol. 2018. Vol. 69. № 5. P. 1088-1098.

36. Waziry R., Hajarizadeh B., Grebely J. et al. Hepatocellular carcinoma risk following direct-acting antiviral HCV therapy: a systematic review, meta-analyses, and meta-regression // J. Hepatol. 2017. Vol. 67. № 6. P. 1204-1212.

37. Mariño Z., Darnell A., Lens S. et al. Time association between hepatitis C therapy and hepatocellular carcinoma emergence in cirrhosis: relevance of non-characterized nodules // J. Hepatol. 2019. Vol. 70. № 5. P. 874-884.

38. Reig M., Mariño Z., Perelló C. et al. Unexpected high rate of early tumor recurrence in patients with HCV-related HCC undergoing interferon-free therapy // J. Hepatol. 2016. Vol. 65. № 4. P. 719-726.

39. Cheung M.C.M., Walker A.J., Hudson B.E. et al. Outcomes after successful direct-acting antiviral therapy for patients with chronic hepatitis $C$ and decompensated cirrhosis // J. Hepatol. 2016. Vol. 65. № 4. P. 741-747.

40. Singal A.G., Rich N.E., Mehta N. et al. Direct-acting antiviral therapy not associated with recurrence of hepatocellular carcinoma in a multicenter North American cohort study // Gastroenterology. 2019. Vol. 156. № 6. P. 1683-1692.e1.

41. Nagata H., Nakagawa M., Asahina Y. et al. Ochanomizu Liver Conference Study Group. Effect of interferon-based and -free therapy on early occurrence and recurrence of hepatocellular carcinoma in chronic hepatitis $\mathrm{C} / /$ J. Hepatol. 2017. Vol. 67. № 5. P. 933-939.

42. Huang A.C., Mehta N., Dodge J.L. et al. Direct-acting antivirals do not increase the risk of hepatocellular carcinoma recurrence after local-regional therapy or liver transplant waitlist dropout // Hepatology. 2018. Vol. 68. № 2. P. 449-461.

43. Heimbach J.K., Kulik L.M., Finn R.S. et al. AASLD guidelines for the treatment of hepatocellular carcinoma // Hepatology. 2018. Vol. 67. № 1. P. 358-380.

44. Farhang Zangneh H., Wong W.W.L., Sander B. et al. Cost effectiveness of hepatocellular carcinoma surveillance after a sustained virologic response to therapy in patients with hepatitis $\mathrm{C}$ virus infection and advanced fibrosis // Clin.

Gastroenterol. Hepatol. 2019. Vol. 17. № 9. P. 1840-1849.e16. 
45. D'Ambrosio R., Aghemo A., Rumi M.G. et al. Persistence of hepatocellular carcinoma risk in hepatitis C patients with a response to IFN and cirrhosis regression // Liver Int. 2018. Vol. 38. № 8. P. 1459-1467.

46. Ioannou G.N., Green P.K., Berry K. HCV eradication induced by direct-acting antiviral agents reduces the risk of hepatocellular carcinoma // J. Hepatol. 2017. pii:S0168-8278(17)32273-0.

47. Degasperi E., D'Ambrosio R., Iavarone M. et al. Factors associated with increased risk of de novo or recurrent hepatocellular carcinoma in patients with cirrhosis treated with direct-acting antivirals for HCV infection // Clin. Gastroenterol. Hepatol. 2019. Vol. 17. № 6. P. 1183-1191.e7.

48. Lleo A., Aglitti A., Aghemo A. et al. Predictors of hepatocellular carcinoma in HCV cirrhotic patients treated with direct acting antivirals // Dig. Liver Dis. 2019. Vol. 51. № 2. P. 310-317.

49. Meringer H., Shibolet O., Deutsch L. Hepatocellular carcinoma in the post-hepatitis C virus era: should we change the paradigm? // World J. Gastroenterol. 2019. Vol. 25. № 29. P. 3929-3940.

50. Calvaruso V., Cabibbo G., Cacciola I. et al. Incidence of hepatocellular carcinoma in patients with HCV-associated cirrhosis treated with direct-acting antiviral agents // Gastroenterology. 2018. Vol. 155. № 2. P. 411-421.e4.

51. Chen C.L., Yang H.I., Yang W.S. et al. Metabolic factors and risk of hepatocellular carcinoma by chronic hepatitis B/C infection: a follow-up study in Taiwan // Gastroenterology. 2008. Vol. 135. № 2. P. 111-121.

52. Kishta S., Tabll A., Omanovic Kolaric T. et al. Risk factors contributing to the occurrence and recurrence of hepatocellular carcinoma in hepatitis $\mathrm{C}$ virus patients treated with direct-acting antivirals // Biomedicines. 2020. Vol. 8. № 6. P. 175.

53. Orci L.A., Sanduzzi-Zamparelli M., Caballol B. et al. Incidence of hepatocellular carcinoma in patients with non-alcoholic fatty liver disease: a systematic review, meta-analysis, and meta-regression // Clin. Gastroenterol. Hepatol. 2021. S1542-3565(21)00505-X.

54. Valenti L., Al-Serri A., Daly A.K. et al. Homozygosity for the patatin-like phospholipase-3/adiponutrin I148M polymorphism influences liver fibrosis in patients with nonalcoholic fatty liver disease // Hepatology. 2010. Vol. 51. № 4. P. 1209-1217.

55. Liu Y.L., Patman G.L., Leathart J.B. et al. Carriage of the PNPLA3 rs738409 C \&gt;G polymorphism confers an increased risk of non-alcoholic fatty liver disease associated hepatocellular carcinoma // J. Hepatol. 2014. Vol. 61. № 1. P. 75-81.

56. Liu Y.L., Reeves H.L., Burt A.D. et al. TM6SF2 rs58542926 influences hepatic fibrosis progression in patients with non-alcoholic fatty liver disease // Nat. Commun. 2014. Vol. 5. P. 4309.

57. Koo B.K., Joo S.K., Kim D. et al. Additive effects of PNPLA3 and TM6SF2 on the histological severity of non-alcoholic fatty liver disease // J. Gastroenterol. Hepatol. 2018. Vol. 33. № 6. P. 1277-1285.

58. Walker A.J., Peacock C.J., Pedergnana V. et al. Host genetic factors associated with hepatocellular carcinoma in patients with hepatitis C virus infection: a systematic review // J. Viral. Hepat. 2018. Vol. 25. № 5. P. 442-456.

59. Mittal S., El-Serag H.B., Sada Y.H. et al. Hepatocellular carcinoma in the absence of cirrhosis in United States veterans is associated with nonalcoholic fatty liver disease // Clin. Gastroenterol. Hepatol. 2016. Vol. 14. № 1. P. 124-131.e1.

60. Bolondi L. Screening for hepatocellular carcinoma in cirrhosis // J. Hepatol. 2003. Vol. 39. № 6. P. 1076-1084.

61. Simmons O., Fetzer D.T., Yokoo T. et al. Predictors of adequate ultrasound quality for hepatocellular carcinoma surveillance in patients with cirrhosis // Aliment. Pharmacol. Ther. 2017. Vol. 45. № 1. P. 169-177.

62. European Association for the Study of the Liver. EASL Clinical Practice Guidelines. Management of hepatocellular carcinoma // J. Hepatol. 2018. Vol. 69. № 1. P. 182-236.

\title{
Risk Factors and Hepatocellular Cancer Screening After Hepatitis C Virus Eradication
}

\author{
A.O. Bueverov, PhD, Prof., ${ }^{1,2}$, P.O. Bogomolov, $\mathrm{PhD}^{2}$, V.E. Syutkin, $\mathrm{PhD}^{3,4}$ \\ ${ }^{1}$ I.M. Sechenov First Moscow State Medical University \\ ${ }^{2}$ Moscow Regional Research Clinical Institute named after M.F. Vladimirsky \\ ${ }^{3}$ Federal Medical and Biological Agency of Russia \\ ${ }^{4}$ Sklifosovsky Research Institute of Emergency Medicine
}

Contact person: Alexey O. Bueverov, bcl72@yandex.ru

Hepatocellular cancer (HCC) usually develops against the background of chronic liver disease. Until recently, the most common etiology of HCC was infection with hepatitis $C$ virus (HCV). The appearance of direct-acting antiviral drugs (DAAD) has become a big breakthrough in the treatment of HCV infection. A stable virological response can now be achieved in almost all treated patients, even in people at high risk of HCC, primarily with cirrhosis of the liver. At the same time, reports gradually began to accumulate about the continued risk of malignant transformation after successful therapy of DAAD. Simultaneously with the decrease in the burden of cirrhosis caused by HCV, the etiological role of non-alcoholic fatty liver disease (NAFLD) has sharply increased. Moreover, in a significant part of patients with NAFLD, HCC is formed at the pre-cirrhotic stage. These changes in the etiology and epidemiology of HCC suggest the revision of patient management tactics.

Key words: hepatocellular cancer, hepatitis $C$ virus, risk factors, direct-acting antiviral drugs, non-alcoholic fatty liver disease 